\title{
Effect of disorder on the pressure-induced superconducting state of $\mathrm{CeAu}_{2} \mathrm{Si}_{2}$
}

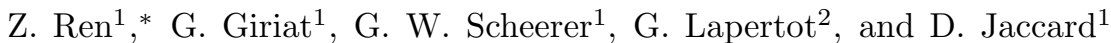 \\ ${ }^{1}$ DQMP - University of Geneva, 24 Quai Ernest-Ansermet, 1211 Geneva 4, Switzerland and \\ ${ }^{2}$ SPSMS, UMR-E CEA/UJF-Grenoble 1, INAC, Grenoble, F-38054, France
}

(Dated: September 20, 2018)

\begin{abstract}
$\mathrm{CeAu}_{2} \mathrm{Si}_{2}$ is a newly discovered pressure-induced heavy fermion superconductor which shows very unusual interplay between superconductivity and magnetism under pressure. Here we compare the results of high-pressure measurements on single crystalline $\mathrm{CeAu}_{2} \mathrm{Si}_{2}$ samples with different levels of disorder. It is found that while the magnetic properties are essentially sample independent, superconductivity is rapidly suppressed when the residual resistivity of the sample increases. We show that the depression of bulk $T_{\mathrm{c}}$ can be well understood in terms of pair breaking by nonmagnetic disorder, which strongly suggests an unconventional pairing state in pressurized $\mathrm{CeAu}_{2} \mathrm{Si}_{2}$. Furthermore, increasing the level of disorder leads to the emergence of another phase transition at $T^{*}$ within the magnetic phase, which might be in competition with superconductivity.
\end{abstract}

PACS numbers: 74.62.Fj, 74.62.En, 74.70.Tx

\section{INTRODUCTION}

Ce-based magnetic compounds that become superconducting under pressure have attracted a lot of attention because of the intimate connection between superconductivity (SC) and magnetic or valence instabilities [1]. Prominent examples include $\mathrm{CeCu}_{2} \mathrm{Ge}_{2}$ [2], $\mathrm{CePd}_{2} \mathrm{Si}_{2}$ [3], $\mathrm{CeIn}_{3}$ [4] and $\mathrm{CeRhIn}_{5}$ [5]. Very recently, pressure-induced heavy fermion $\mathrm{SC}$ with transition temperatures $T_{\mathrm{c}}$ up to $2.5 \mathrm{~K}$ is observed in the antiferromagnet $\mathrm{CeAu}_{2} \mathrm{Si}_{2}$ [6], which is both isostructural and isoelectronic to the first unconventional superconductor $\mathrm{CeCu}_{2} \mathrm{Si}_{2}$ [7]. It is quite remarkable in $\mathrm{CeAu}_{2} \mathrm{Si}_{2}$ that $\mathrm{SC}$ coexists with long-range magnetic order over a huge pressure interval of $11 \mathrm{GPa}$. Moreover, in approximately one-third of this pressure range, the magnetic ordering temperature $T_{\mathrm{M}}$ and $T_{\mathrm{c}}$ are simultaneously enhanced by pressure [6]. These behaviors are hardly explained within the common scenarios of Cooper pairing mediated by spin [8] or valence-fluctuations 9], and thus it is of particular interest to clarify the nature of SC in this material.

The $T_{\mathrm{c}}$ response to the level of nonmagnetic disorder is known to provide useful information for the phase of the superconducting gap function. For conventional $s$-wave superconductors, no pair breaking is expected by nonmagnetic disorder as long as the system remains metallic, according to the Anderson's theorem [10]. By contrast, for non $s$-wave superconductors, in which there is a sign reversal in the superconducting gap function, scattering from nonmagnetic disorder averages out the gap over the Fermi surface and results in a strong suppression of $T_{\mathrm{c}}$. This effect has been observed in a number of unconventional superconductors, such as $\mathrm{UPt}_{3}$ [11, $\mathrm{YBaCu}_{3} \mathrm{O}_{6+x}$ 12], $\mathrm{Sr}_{2} \mathrm{RuO}_{4}$ [13], BEDT-TTF salts [14] and $\mathrm{CePt}_{3} \mathrm{Si}$ [15]. Although it is commonly believed that the pairing symmetry is non $s$-wave for Ce-based pressure-induced superconductors [16], there is little systematic study of a similar effect at high pressure.
In this paper, we present the pressure responses of two $\mathrm{CeAu}_{2} \mathrm{Si}_{2}$ crystals grown from different fluxes with inplane residual resistivities $\rho_{0}=1.8$ and $12.2 \mu \Omega \mathrm{cm}$, respectively. The results show that while the critical pressures for the disappearance of magnetism and the delocalization of the Ce $4 f$ electrons are almost independent on $\rho_{0}$, the high- $\rho_{0}$ sample shows a much narrower pressure range for $\mathrm{SC}$ and a considerably lower maximum $T_{\mathrm{c}}$. A detailed analysis indicates that at $\sim 21.2 \mathrm{GPa}, \mathrm{SC}$ with an initial onset $T_{\mathrm{c}}$ of $\sim 2.5 \mathrm{~K}$ is destroyed when $\rho_{0}$ exceed $\sim 46 \mu \Omega \mathrm{cm}$, i.e. when the carrier mean free path is reduced to be similar to the superconducting coherence length. Since there is good evidence that $\rho_{0}$ is dominated by the contribution of nonmagnetic disorder, our results point to unconventional $\mathrm{SC}$ in $\mathrm{CeAu}_{2} \mathrm{Si}_{2}$ under pressure. In addition, the high- $\rho_{0}$ sample displays another phase transition at a temperature below $T_{\mathrm{M}}$, which is probably competing with SC.

\section{EXPERIMENTAL}

Crystal growth of $\mathrm{CeAu}_{2} \mathrm{Si}_{2}$ samples by $\mathrm{Sn}$ flux and $\mathrm{Au}-\mathrm{Si}$ self-flux are described in detail in Ref. [6]. The resulting crystals are labeled hereafter as $\mathrm{CeAu}_{2} \mathrm{Si}_{2}(\mathrm{Sn})$ and $\mathrm{CeAu}_{2} \mathrm{Si}_{2}$ (self), respectively. Within the resolution limits of x-ray and microprobe techniques, no difference is observed in the crystal structure and chemical composition of the Sn- and self-flux samples.

High pressure experiments were performed using a Bridgman-type sintered diamond-anvil cell with steatite as soft-solid pressure medium and lead $(\mathrm{Pb})$ as pressure gauge [17]. The results of high-pressure experiments on $\mathrm{CeAu}_{2} \mathrm{Si}_{2}(\mathrm{Sn})$ have been reported in Ref. [6]. For $\mathrm{CeAu}_{2} \mathrm{Si}_{2}$ (self), measurements are carried out in two different pressure cells. In the first pressure cell, only resistivity is measured up to $25.5 \mathrm{GPa}$. The second pressure cell is designed to measure both resistivity and ac heat capacity, but the pressure is limited to $20.5 \mathrm{GPa}$. In 


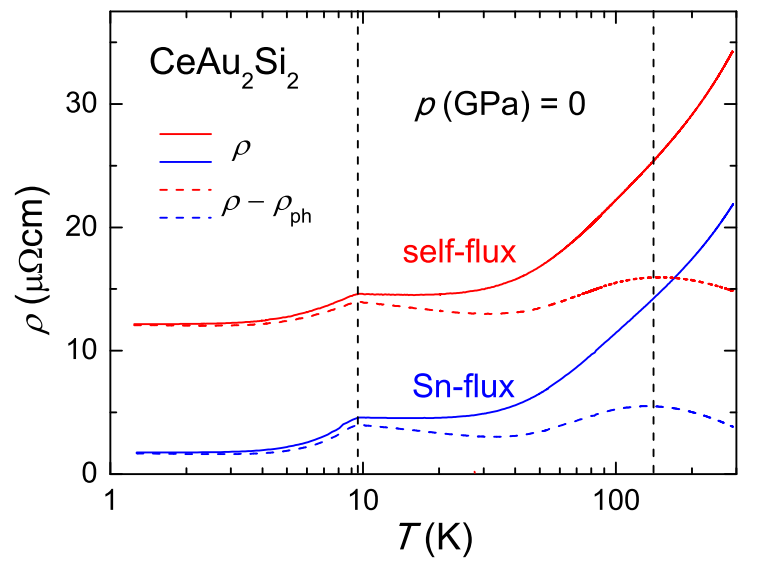

FIG. 1: (Color online) Logarithmic temperature dependence of the in-plane resistivity at ambient pressure before (solid lines) and after (dashed lines) subtraction of the phonon contribution for the $\mathrm{CeAu}_{2} \mathrm{Si}_{2}$ crystals grown from the different fluxes. The vertical dotted lines are a guide to the eyes.

both cells, the $\mathrm{CeAu}_{2} \mathrm{Si}_{2}$ (self) sample with its $a b$ plane perpendicular to the compressive force is connected in series with the $\mathrm{Pb}$ gauge. The resistivity was measured by using a standard four-probe method. For ac-calorimetry measurements, a chromel wire, which is otherwise used as a voltage lead, serves as the heater, and the sample temperature oscillations are detected by a $\mathrm{Au} / \mathrm{AuFe}(0.07 \%)$ thermocouple. More details of the measurement procedure and data analysis can be found in Ref. [17]. A good agreement is found between the results of the two cells, indicating that they reflect the intrinsic properties of the sample.

\section{RESULTS AND DISCUSSION}

\section{A. Ambient pressure results}

Figure 1 shows the comparison of the ambient pressure resistivity data of the $\mathrm{CeAu}_{2} \mathrm{Si}_{2}$ crystals grown from the different fluxes. It can be observed that the resistivity curve of $\mathrm{CeAu}_{2} \mathrm{Si}_{2}$ (self) is an almost rigid upshift of that of $\mathrm{CeAu}_{2} \mathrm{Si}_{2}(\mathrm{Sn})$. After subtraction of the phonon contribution to the resistivity $\left(\rho_{\mathrm{ph}}\right)$, which is assumed to be linear in temperature and pressure independent, both samples exhibit a resistivity maximum at $\sim 140 \mathrm{~K}$ and a sharp drop in resistivity due to the magnetic ordering below $T_{N} \approx 10 \mathrm{~K}$. Furthermore, $\rho_{\mathrm{ph}} \approx 0.067 T(\mu \Omega \mathrm{cm})$ estimated for $\mathrm{CeAu}_{2} \mathrm{Si}_{2}$ (self) is in agreement with that of $\mathrm{CeAu}_{2} \mathrm{Si}_{2}(\mathrm{Sn})$ [6] within the geometrical factor uncertainty $(\sim 10 \%)$. Hence the resistivities of the two crystals differ only by their $\rho_{0}$ values, which correspond to different degrees of disorder.

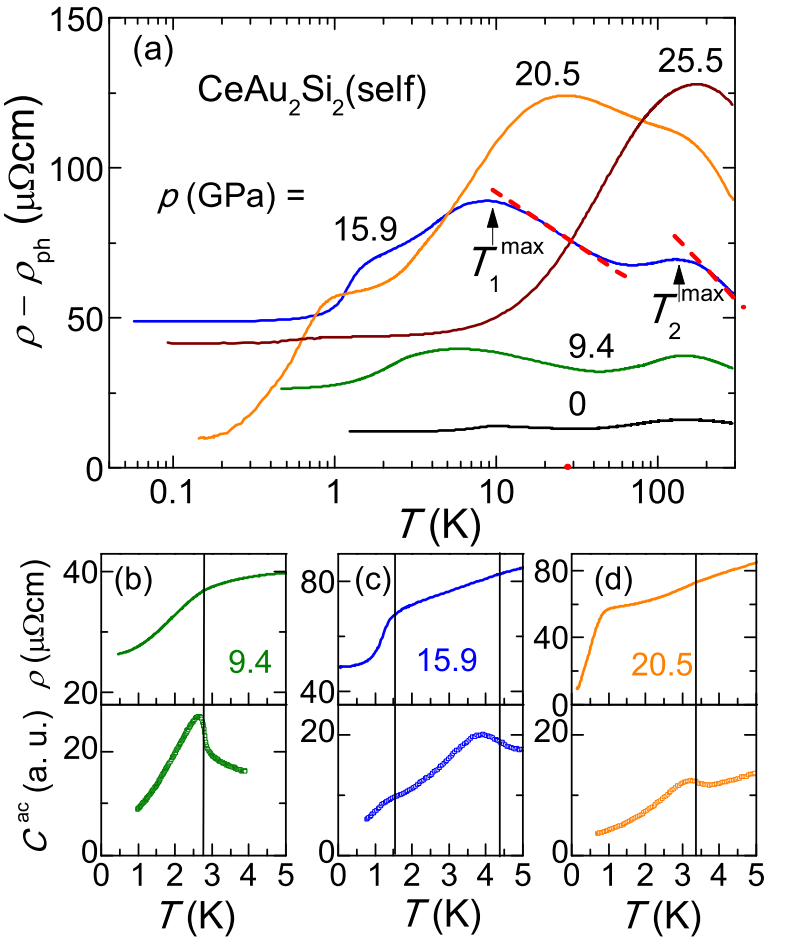

FIG. 2: (Color online) (a) For typical pressures, logarithmic temperature dependence of in-plane resistivity of the self-flux grown $\mathrm{CeAu}_{2} \mathrm{Si}_{2}$ (self) crystals after subtraction of the phonon contribution. The two characteristic maxima $T_{1}^{\max }$ and $T_{2}^{\max }$ at $15.9 \mathrm{GPa}$ are marked by arrows. The dashed lines are a guide to the eyes, evidencing the $-\ln T$ behavior. Panels (b) - (d) show the comparison of the resistivity and ac heat capacity for three different pressures. The solid lines are a guide to the eyes.

\section{B. Pressure response of $\mathrm{CeAu}_{2} \mathrm{Si}_{2}$ (self)}

Typical results at selected pressures of the resistivity $(\rho)$ and ac heat capacity $\left(C^{\mathrm{ac}}\right)$ of $\mathrm{CeAu}_{2} \mathrm{Si}_{2}$ (self) are shown in Fig. 2. Apart from a much larger residual resistivity $\rho_{0}$, the overall behavior of the non-phononic resistivity [Fig. 2(a)] is very similar to that of $\mathrm{CeAu}_{2} \mathrm{Si}_{2}(\mathrm{Sn})$. At the intermediate pressure of $15.9 \mathrm{GPa}$, two broad maxima exist at $T_{1}^{\max }$ and $T_{2}^{\max }$, and above each maxima the data follow a $-\ln T$ dependence, which manifests the incoherent Kondo scattering of the ground state and excited crystal-filed (CF) levels, respectively [19, 20]. As pressure is increased to $20.5 \mathrm{GPa}, T_{1}^{\max }$ almost doubles while $T_{2}^{\max }$ remains nearly unchanged. At the highest pressure of $25.5 \mathrm{GPa}$, the Kondo effect dominates over the CF splitting so that the two maxima are already merged into a single peak at $\sim 180 \mathrm{~K}$. Concomitantly, both the magnitude of the resistivity and the $-\ln T$ slope increase rapidly with pressure, signifying a strong enhancement of the Kondo interaction under pressure.

In Fig. 2(b)-(d), we compare the results of $\rho$ and $C^{\text {ac }}$ below $5 \mathrm{~K}$ at three typical pressures. At $9.4 \mathrm{GPa}$, the 


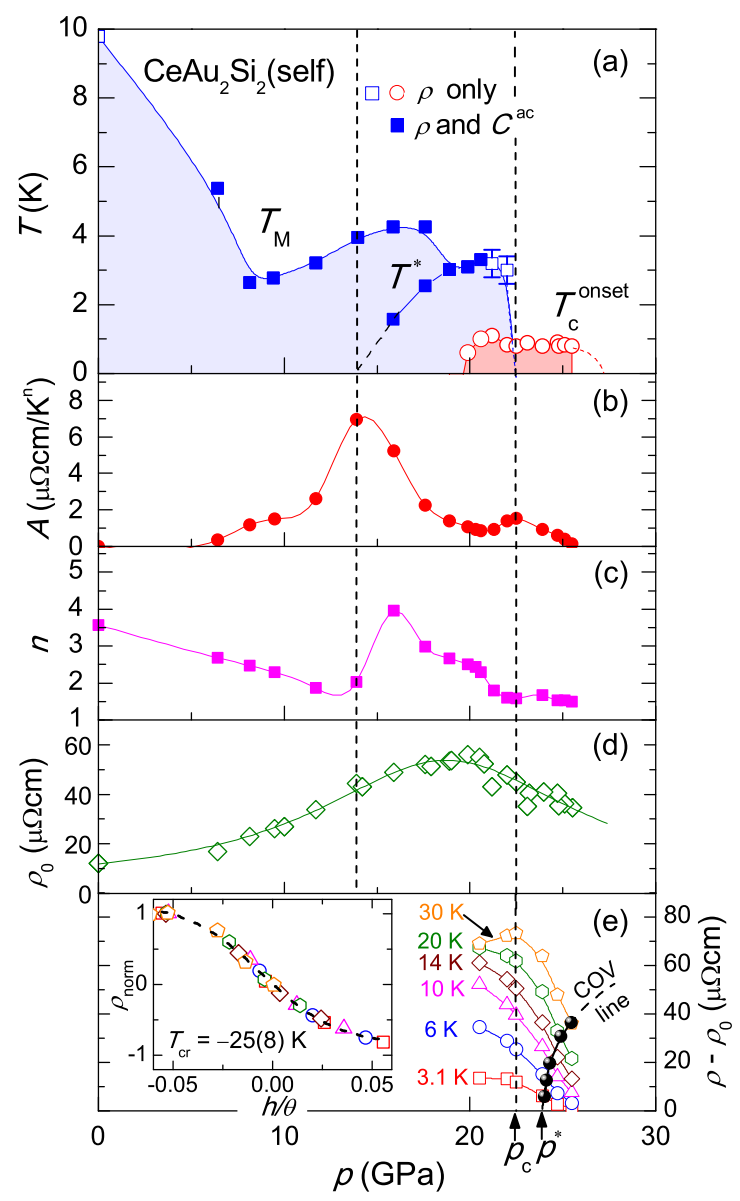

FIG. 3: (Color online) (a) Experimental $p$ - $T$ phase diagram of $\mathrm{CeAu}_{2} \mathrm{Si}_{2}$ (self). $T_{\mathrm{c}}^{\mathrm{onset}}$ and $T_{\mathrm{M}}$ represent the superconducting transition onset and the magnetic ordering temperatures, respectively. The open (closed) symbols denote the data extracted from the resistivity measurements only (both resistivity and heat capacity measurements). (b), (c) and (d) show the pressure dependencies of the coefficient $A$, temperature exponent $n$, and residual resistivity $\rho_{0}$, respectively, obtained from the fitting of the power law $\rho(T)=\rho_{0}+A T^{n}$ to the low temperature resistivity data. (e) Plot of $\rho^{*}=\rho-\rho_{0}$ versus $p$ at selected temperatures up to $30 \mathrm{~K}$. The closed circles indicate for each isotherm the $50 \%$ drop of $\rho^{*}$ compared to its value at $22.5 \mathrm{GPa}$ and define the crossover (COV) line. The inset shows the collapse of all normalized data $\rho_{\text {norm }}$ when plotted as a function of the generalized distance $h / \theta$ from the critical end point located at $p^{*} \approx 23.9 \mathrm{GPa}$ and $T_{\mathrm{cr}}=-25$ $K$. The vertical dashed lines are a guide to the eyes. The two critical pressures $p_{\mathrm{c}}$ and $p^{*}$ are indicated by labeled arrows.

change of slope in resistivity at $\sim 2.7 \mathrm{~K}$ coincides with the midpoint of the sharp jump in $C^{\mathrm{ac}}(T)$, indicating a magnetic ordering [21]. Notably, at $15.9 \mathrm{GPa}$ two jumps in $C^{\text {ac }}(T)$ are observed. The one at $\sim 4.4 \mathrm{~K}$ corresponds to a slight slope change of the resistivity, while the other at $\sim 1.5 \mathrm{~K}$ is accompanied by a steep resistivity drop that is independent of the applied current and is not due to SC. Thus it appears that at this pressure the sample undergoes two successive phase transitions, similarly to $\mathrm{CeCu}_{2} \mathrm{Ge}_{2}$ at $\sim 3 \mathrm{GPa}$ [22]. At $20.5 \mathrm{GPa}$, the highest pressure at which $C^{\text {ac }}$ is measured, only one magnetic transition is detectable in both $\rho$ and $C^{\text {ac }}(T)$ at $3.3 \mathrm{~K}$, and, below $1 \mathrm{~K}$ the incomplete resistive transition without any corresponding anomaly in $C^{\mathrm{ac}}(T)$ indicates $\mathrm{SC}$ of filamentary nature.

The resulting pressure-temperature phase diagram is shown in Fig. 3(a). The magnetic ordering temperature $T_{\mathrm{M}}$ initially decreases with increasing pressure, as expected, due to the enhancement of the Kondo interaction. However, $T_{\mathrm{M}}$ already starts to increase with pressure above $8 \mathrm{GPa}$. At $15.9 \mathrm{GPa}$, another transition appears at $T^{*}<T_{\mathrm{M}}$. With further increasing pressure, $T^{*}$ rises while $T_{\mathrm{M}}$ shows a maximum, and the two transitions merge at $18.9 \mathrm{GPa}$. At higher pressures, $T_{\mathrm{M}}$ exhibits a dome-shaped dependence and finally disappears abruptly above $22.5 \mathrm{GPa}$. On the other hand, SC is observed from $19.9 \mathrm{GPa}$ up to the highest investigated pressure. It is pointed out that zero resistivity is achieved only at 21.2 $\mathrm{GPa}$, the pressure at which the onset $T_{\mathrm{c}}$ reaches its maximum of $\sim 1.1 \mathrm{~K}$. Both $T_{\mathrm{c}}$ and $T_{\mathrm{M}}$ are enhanced within a narrow pressure range between 19.9 and $20.6 \mathrm{GPa}$.

Figure 3(b)-(d) shows the fitting parameters of the power law $\rho(T)=\rho_{0}+A T^{n}$ to the resistivity data plotted as a function of pressure. Thanks to a sufficiently broad temperature window between $T_{\mathrm{c}}$ and $T_{\mathrm{M}}$, we are able to extract reliable parameters for the whole pressure range. The $A$ coefficient exhibits two maxima at 13.9 and 22.5 $\mathrm{GPa}$, respectively. The latter together with a minimum $n$ exponent $(n \approx 1.5)$ coincides with the disappearance of the magnetic order, indicating a magnetic quantum critical point at $p_{\mathrm{c}}=22.5 \pm 0.5 \mathrm{GPa}$. However, the former with $n \approx 2$ occurs at a pressure close to that of the interpolation of $T^{*}$ to $0 \mathrm{~K}$, which points to the possibility of a putative quantum phase transition occurring within the magnetic phase. Actually, $\rho_{0}$ shows a broad peak at $\sim 20 \mathrm{GPa}$, suggesting that the maximum scattering rate happens in between these two QCPs. Nevertheless, the large $A$ value at $13.9 \mathrm{GPa}$ may contain a significant contribution from the electron-magnon scattering, and thus provides little information of the effective mass.

Figure 3(e) shows the plot of isothermal resistivity $\rho^{*}(p)=\rho(p)-\rho_{0}(p)$ versus $p$ at selected temperatures up to $30 \mathrm{~K}$. Above $22.5 \mathrm{GPa}, \rho^{*}(p)$ decreases steeply with pressure, revealing the continuous delocalization of the Ce $4 f$ electrons. For the data analyses, we follow the procedure described in Ref. 23], which is based on the assumption of an underlying critical end point located at $\left(p_{\text {cr }}, T_{\text {cr }}\right)$ in the $p$ - $T$ plane. It turns out that all the normalized resistivity curves $\rho_{\text {norm }}(p)=\left(\rho^{*}(p)\right.$ $\left.-\rho^{*}\left(p_{50 \%}\right)\right) / \rho^{*}\left(p_{50 \%}\right)$ below $30 \mathrm{~K}$ fall on a single curve when plotted against $h / \theta$, where for each temperature, $p_{50 \%}$ denotes the pressure corresponding to the midpoint of the $\rho^{*}(p)$-drop compared to its value at $22.5 \mathrm{GPa}, h$ $=\left(p-p_{50 \%}\right) / p_{50 \%}$ and $\theta=\left(T-T_{\text {cr }}\right) /\left|T_{\text {cr }}\right|$ with the 


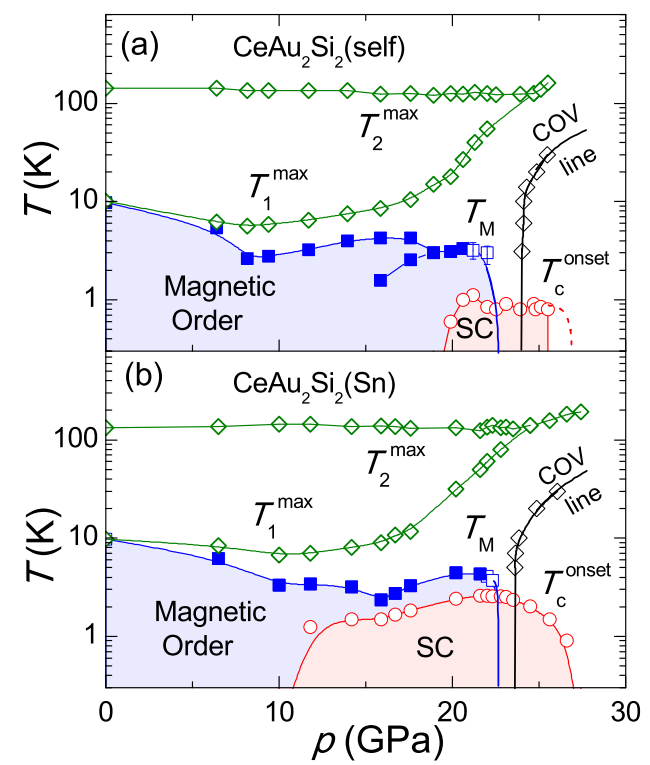

FIG. 4: (Color online) $p-T$ phase diagrams of (a) $\mathrm{CeAu}_{2} \mathrm{Si}_{2}$ (self) and (b) $\mathrm{CeAu}_{2} \mathrm{Si}_{2}(\mathrm{Sn})$ including the temperatures $T_{1}^{\max }$ and $T_{2}^{\max }$. Note that the vertical axis is in logarithmic scale. The crossover(COV) line is determined by the scaling analysis of the resistivity.

only free parameter $T_{\mathrm{cr}}=-25(8) \mathrm{K}$. The negative $T_{\mathrm{cr}}$ indicates a crossover rather than a first-order transition. Moreover, the extrapolation of the temperature dependence of $p_{50 \%}$ to $0 \mathrm{~K}$ yields the critical end pressure $p^{*}(\approx$ $\left.p_{\text {cr }}\right)=23.9 \pm 0.7 \mathrm{GPa}$.

\section{Comparison with the results of $\mathrm{CeAu}_{2} \mathrm{Si}_{2}(\mathrm{Sn})$}

Figure 4 shows the $p-T$ phase diagrams of $\mathrm{CeAu}_{2} \mathrm{Si}_{2}$ (self) and $\mathrm{CeAu}_{2} \mathrm{Si}_{2}(\mathrm{Sn})$ including the lines defined by the temperatures $T_{1}^{\max }$ and $T_{2}^{\max }$ of the resistivity maxima in the paramagnetic phase, as well as the crossover (COV) lines obtained by the $50 \%$ drop of $\rho^{*}$. Clearly, these lines are almost identical for both crystals. Since the temperatures $T_{1}^{\max }$ and $T_{2}^{\max }$ (for $p>15.9 \mathrm{GPa})$ scale approximately with the Kondo temperature and CF splitting energy respectively [20], it is obvious that the pressure evolution of the characteristic high-temperature energy scales are essentially sample independent. This reflects that both the Kondo interaction and CF levels depend mainly on the local environment of the Ce ions.

By contrast, at temperatures below $5 \mathrm{~K}$, the two phase diagrams show significant differences. Although the critical pressures $p_{\mathrm{c}}$ and $p^{*}$ are nearly identical for both samples, in $\mathrm{CeAu}_{2} \mathrm{Si}_{2}$ (self) a much higher pressure (19.9 $\mathrm{GPa}$ ) is needed to induce $\mathrm{SC}$ and the maximum onset $T_{\mathrm{c}}$ is reduced by a factor of 2.3 . As a consequence, the pressure range for the overlap between the magnetic and

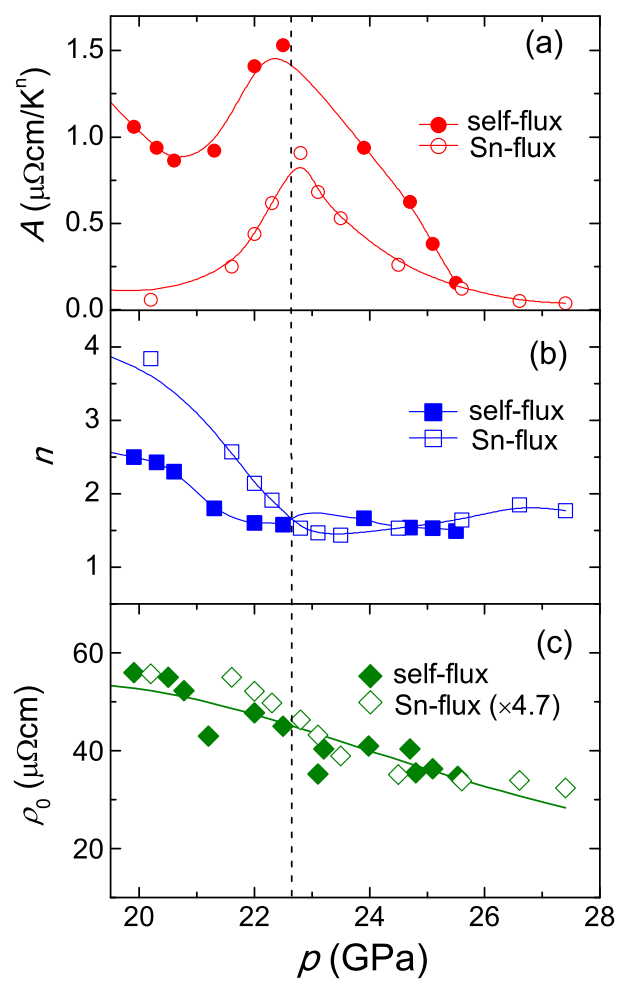

FIG. 5: (Color online) Comparison of the pressure dependencies of the fitting parameters, (a) the $A$ coefficient, (b) the $n$ exponent, (c) the residual resistivity $\rho_{0}$ for $\mathrm{CeAu}_{2} \mathrm{Si}_{2}$ (self) and $\mathrm{CeAu}_{2} \mathrm{Si}_{2}(\mathrm{Sn})$. The open and closed symbols denote the data for $\mathrm{CeAu}_{2} \mathrm{Si}_{2}(\mathrm{Sn})$ and $\mathrm{CeAu}_{2} \mathrm{Si}_{2}$ (self), respectively. Note that the $\rho_{0}$ data for $\mathrm{CeAu}_{2} \mathrm{Si}_{2}(\mathrm{Sn})$ are multiplied by a factor of 4.7. The vertical dashed line is a guide to the eyes.

superconducting phases is restricted to $\sim 3 \mathrm{GPa}$. It is also noteworthy that the pressure evolution of the magnetic order is more complex for $\mathrm{CeAu}_{2} \mathrm{Si}_{2}$ (self). While the origin of the transition at $T^{*}$ remains unclear, it is possible that the resulting ground state is competing with $\mathrm{SC}$, as will be discussed further below.

Figure 5 compares the power-law fitting parameters of the resistivity data above $T_{\mathrm{c}}$ for $\mathrm{CeAu}_{2} \mathrm{Si}_{2}$ (self) and $\mathrm{CeAu}_{2} \mathrm{Si}_{2}(\mathrm{Sn})$. It can be noted that the pressure dependencies of the three parameters are very similar in both cases. The maximum A coefficient and minimum $n(<2)$ exponent at $p_{\mathrm{c}}$ are typical for a QCP. Above $p_{\mathrm{c}}$, while the $n$ values increase only slightly, the $A$ values drop abruptly by more than one order of magnitude, indicating a transition from a strongly to a weakly correlated regime. Moreover, the $\rho_{0}$ data of $\mathrm{CeAu}_{2} \mathrm{Si}_{2}(\mathrm{Sn})$, when multiplied by a factor of 4.7 , match well with those of $\mathrm{CeAu}_{2} \mathrm{Si}_{2}$ (self). This scaling factor is not far from its ambient pressure value $(\sim 6.7)$, suggesting that the difference in the levels of disorder between $\mathrm{CeAu}_{2} \mathrm{Si}_{2}$ (self) and $\mathrm{CeAu}_{2} \mathrm{Si}_{2}(\mathrm{Sn})$ does not change much with pressure.

We next turn our attention to the scaling behavior of the resistivity near $p^{*}$. Figure 6(a) shows the tem- 

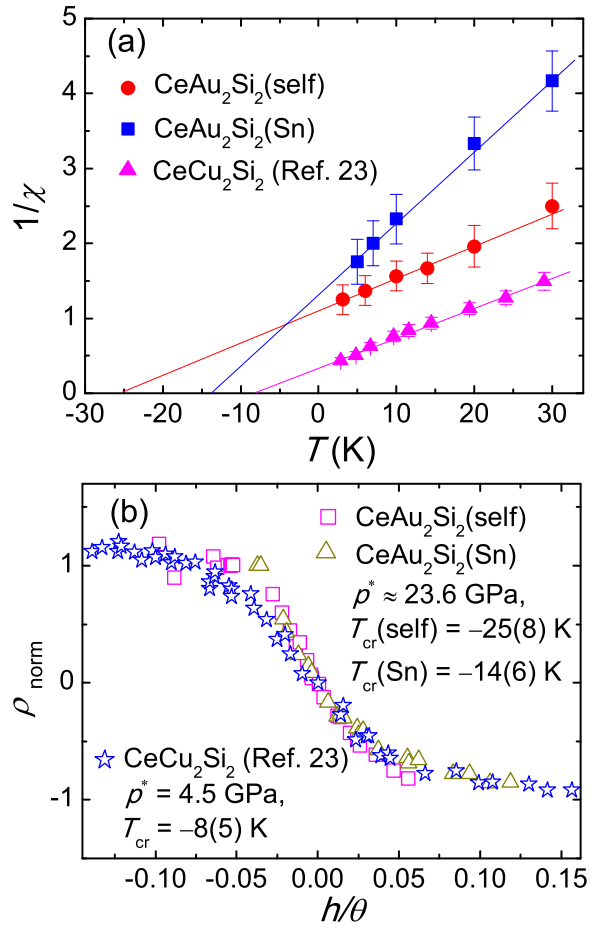

FIG. 6: (Color online) (a) Temperature dependencies of the inverse slope $1 / \chi$ (see text for details) for $\mathrm{CeAu}_{2} \mathrm{Si}_{2}$ (self), $\mathrm{CeAu}_{2} \mathrm{Si}_{2}(\mathrm{Sn})$, and $\mathrm{CeCu}_{2} \mathrm{Si}_{2}$ from Ref. 23]. $T_{\text {cr values are }}$ extracted from linear fits to the data (solid lines). (b) $\rho_{\text {norm }}$ data plotted as a function of $h / \theta$ for the three cases, showing a good agreement.

perature dependence of $1 / \chi$, where $\chi=\left|d \rho_{\text {norm }} / d p\right|_{p_{50 \%}}$ is the slope of the resistivity drop at the midpoint, for $\mathrm{CeAu}_{2} \mathrm{Si}_{2}$ (self) and $\mathrm{CeAu}_{2} \mathrm{Si}_{2}(\mathrm{Sn})$ [6], as well as $\mathrm{CeCu}_{2} \mathrm{Si}_{2}$ [23] for comparison. In the three cases, $1 / \chi$ diminishes on lowering temperature, indicating that the slope becomes increasingly steep as the systems approach their critical end point located at $\left(p_{\mathrm{cr}}, T_{\mathrm{cr}}\right)$. Assuming $1 / \chi \propto\left(T-T_{\text {cr }}\right), T_{\text {cr }}$ can be obtained by a linear extrapolation of the data to the $x$-axis. Remarkably, despite the large differences in $T_{\text {cr }}$ ranging from $-25 \mathrm{~K}$ to -8 $\mathrm{K}$, the $\rho_{\text {norm }}$ data below $30 \mathrm{~K}$ follow almost the same curve when plotted as a function of $h / \theta$ especially for $h / \theta>0\left(p>p_{50 \%}\right)$, as shown in Fig. 6(b). This means that, for a generalized distance $h / \theta$ from the critical end point, $\rho_{\text {norm }}$ behaves in the same way for both $\mathrm{CeAu}_{2} \mathrm{Si}_{2}$ and $\mathrm{CeCu}_{2} \mathrm{Si}_{2}$. Further study is needed to establish the level of generality of such a behavior in related Ce-based compounds. For our two $\mathrm{CeAu}_{2} \mathrm{Si}_{2}$ crystals, it appears that higher (less negative) $T_{\text {cr }}$ corresponds to higher $T_{\mathrm{c}}$. However, despite their similar $T_{\mathrm{c}}$ values near their respective $p^{*}$, the absolute $T_{\text {cr }}$ value of $\mathrm{CeAu}_{2} \mathrm{Si}_{2}(\mathrm{Sn})$ is nearly twice that of $\mathrm{CeCu}_{2} \mathrm{Si}_{2}$. Nevertheless, the $T_{\text {cr }}$ value of $\mathrm{CeAu}_{2} \mathrm{Si}_{2}$ could be considerably underestimated due to the unavoidable degradation of hydrostatic conditions at very high pressure.
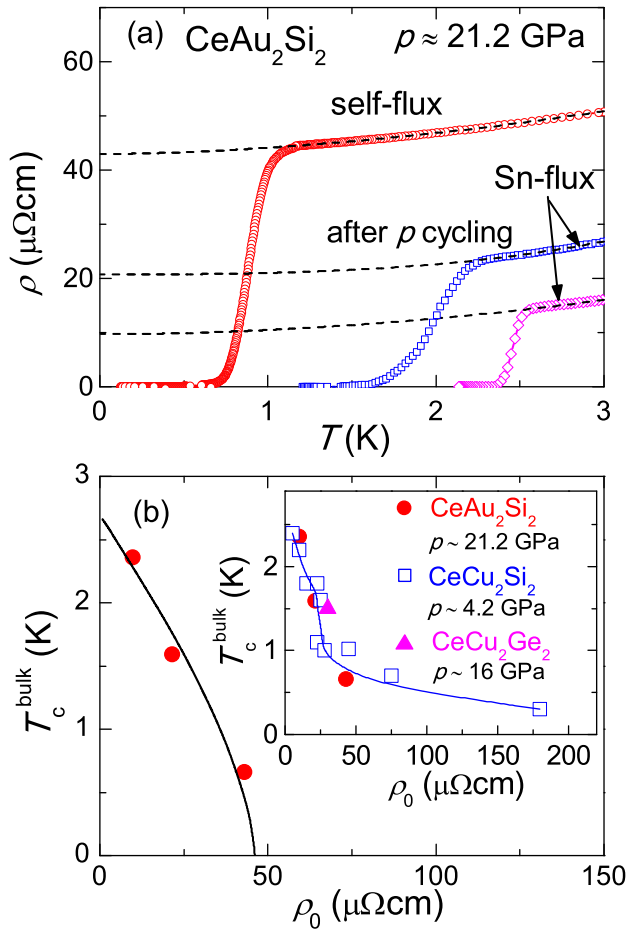

FIG. 7: (Color online) (a) Temperature dependencies of the resistivity of $\mathrm{CeAu}_{2} \mathrm{Si}_{2}$ at $\sim 21.2 \mathrm{GPa}$ for different level of disorder. The dashed lines denote the power-law fit employed to extract the residual resistivity $\rho_{0}$. (b) Plot of $T_{\mathrm{c}}^{\mathrm{bulk}}$ versus $\rho_{0}$ for $\mathrm{CeAu}_{2} \mathrm{Si}_{2}$ at $\sim 21.2 \mathrm{GPa}$. The solid line is a fit from the AG theory to the data. The inset shows $T_{\mathrm{c}}^{\mathrm{bulk}}$ plotted as a function of $\rho_{0}$ for $\mathrm{CeAu}_{2} \mathrm{Si}_{2}$ at $\sim 21.2 \mathrm{GPa}, \mathrm{CeCu}_{2} \mathrm{Si}_{2}$ at $\sim 4.2 \mathrm{GPa}$ and $\mathrm{CeCu}_{2} \mathrm{Ge}_{2}$ at $\sim 16 \mathrm{GPa}$. Note that we have collected all available data from Refs. [9, 18, 22 25, 33].

To gain insight into the pressure-induced $\mathrm{SC}$ in $\mathrm{CeAu}_{2} \mathrm{Si}_{2}$, we show in Fig. 7(a) the resistivity below $3 \mathrm{~K}$ of the Sn- and self-flux grown crystals around 21.2 $\mathrm{GPa}$, a pressure close to that of the optimum $T_{c}$. As can be seen, $\mathrm{CeAu}_{2} \mathrm{Si}_{2}(\mathrm{Sn})$ has a low $\rho_{0}$ and shows a sharp superconducting transition below $2.55 \mathrm{~K}$ with a width of $0.18 \mathrm{~K}$. By contrast, $\mathrm{CeAu}_{2} \mathrm{Si}_{2}$ (self) has a much higher $\rho_{0}$, and its superconducting onset temperature is shifted to $1.15 \mathrm{~K}$ while the transition width increases to $0.55 \mathrm{~K}$. This trend is further corroborated by investigating the effect of pressure cycling on $\mathrm{CeAu}_{2} \mathrm{Si}_{2}(\mathrm{Sn})$, which tends to induce further disorder(dislocation) and therefore increase $\rho_{0}$. Indeed, when pressure is increased again up to $\sim 21.2 \mathrm{GPa}$ (after a partial depressurization from 27.6 down to $10 \mathrm{GPa}$ ), the $\rho_{0}$ value doubles while the onset $T_{\mathrm{c}}$ decreases to $\sim 2.2 \mathrm{~K}$. Concomitantly, the resistive transition becomes much broader most likely due to the decrease of pressure homogeneity, which is inevitable after the pressure cycling.

In Fig. 7(b), we plot the dependence of $T_{\mathrm{c}}^{\mathrm{bulk}}$ on $\rho_{0}$ at $\sim 21.2 \mathrm{GPa}$. Here $T_{\mathrm{c}}^{\mathrm{bulk}}$ is defined as the temperature where zero resistivity is achieved, given that the com- 
pleteness of resistive transition coincides with the jump in the ac heat capacity [6]. As can be seen, $T_{\mathrm{c}}^{\text {bulk }}$ decreases rapidly with increasing $\rho_{0}$. For $f$-electron systems, $\rho_{0}$ can be expressed as $\rho_{0}=\rho_{0}^{\text {Born }}+\rho_{0}^{\text {unit }}$, where $\rho_{0}^{\text {Born }}$ and $\rho_{0}^{\text {unit }}$ are due to the nonmagnetic disorder of non- $f$ elements and the defects of Ce ions, respectively [24]. Under pressure, $\rho_{0}^{\text {unit }}$ remains essentially unaffected while $\rho_{0}^{\text {Born }}$ is subject to a large enhancement due to critical fluctuations [26]. The values of $\rho_{0}$ for both $\mathrm{CeAu}_{2} \mathrm{Si}_{2}$ (self) and $\mathrm{CeAu}_{2} \mathrm{Si}_{2}(\mathrm{Sn})$ are much larger at $\sim 21.2 \mathrm{GPa}$ than at ambient pressure, indicating that $\rho_{0}^{\text {Born }}$ dominates $\rho_{0}$. Therefore, our results are consistent with pair breaking by nonmagnetic disorder.

According to the Abrikosov-Gor'kov (AG) theory [27] generalized for nonmagnetic disorder in a non- $s$-wave superconductor 28 30], the suppression of $T_{\mathrm{c}}$ follows $\ln \left(\frac{T_{\mathrm{c} 0}}{T_{\mathrm{c}}}\right)=\Psi\left(\frac{1}{2}+\frac{\alpha T_{\mathrm{c} 0}}{T_{\mathrm{c}}}\right)-\Psi\left(\frac{1}{2}\right)$, where $\Psi$ is the digamma function, $\alpha=\hbar /\left(2 \pi k_{\mathrm{B}} \tau T_{\mathrm{c} 0}\right), \tau$ is the scattering time, and $T_{\mathrm{c} 0}$ is $T_{\mathrm{c}}$ in the disorder-free limit $(\alpha \rightarrow 0)$. The model predicts that $T_{\mathrm{c}}$ vanishes at a critical $\alpha$, which is roughly equivalent to the fact that the carrier mean free path $l$ is comparable to the superconducting (Pippard) coherence length $\xi_{0}$. It turns out that the experimental data obey well the functional form of the AG theory, with $T_{\mathrm{c} 0}^{\mathrm{bulk}} \approx$ $2.7 \mathrm{~K}$ and a critical $\rho_{0}^{\mathrm{cr}} \sim 46 \mu \Omega \mathrm{cm}$. The corresponding critical mean free path $l^{\text {cr }}$ can be estimated using the relation $l=\left(1.27 \times 10^{4}\right) / \rho_{0}(N / V)^{2 / 3}$, where $\rho_{0}$ is in $\Omega \mathrm{cm}$, $N$ is the number of conduction electrons per unit cell, and $V$ is the unit cell volume in $\mathrm{cm}^{3}$ 31]. Assuming $N=6$ (there are two formula units per unit cell) and with $V \approx$ $1.6 \times 10^{-22} \mathrm{~cm}^{3}[6]$, we obtain $l^{\text {cr }} \approx 27 \AA$, which is half of the Ginzburg-Landau(GL) coherence length $\xi_{\mathrm{GL}}(0)=$ $55 \AA\left(\approx \xi_{0}\right)$ deduced from the measurement of the upper critical field at $22.3 \mathrm{GPa}$ with $T_{\mathrm{c}} \sim 2.5 \mathrm{~K}[6]$. It should be pointed out that $l^{\text {cr }}$ could be underestimated due to the following reasons: (i) The $N$ value is overestimated; (ii) Only the parts of the sample with a low enough $\rho_{0}$ become superconducting such that $\rho_{0}^{\text {cr }}$ is overestimated; (iii) The calculated $l^{\text {cr }}$ reflects mainly the contribution from the scattering of the light quasiparticles, while $l^{\text {cr }}$ for the heavy quasiparticles that form Cooper pairs could be longer [32]. Thus it is reasonable to speculate that the actual $l^{\text {cr }}$ is close to $\xi_{0}$.

The above analysis shows that the suppression of bulk $T_{\mathrm{c}}$ can be understood, not only qualitatively but also quantitatively, within the pair breaking model. This strongly points to unconventional pairing in the superconducting state of $\mathrm{CeAu}_{2} \mathrm{Si}_{2}$ under pressure. As a matter of fact, in $\mathrm{CeCu}_{2} \mathrm{Si}_{2}$ at a similar volume $V(p \sim 4.2$ GPa) [6], the $\rho_{0}$ dependence of the maximum $T_{\mathrm{c}}^{\text {bulk }}$ shows very similar behavior to that of $\mathrm{CeAu}_{2} \mathrm{Si}_{2}$ for $\rho_{0}<50$ $\mu \Omega \mathrm{cm}$ [inset of Fig. 7(b)] [9, 18, 22 25, 33], suggesting a common mechanism of SC in these compounds. Moreover, at this $V$ magnetic order is still present in $\mathrm{CeAu}_{2} \mathrm{Si}_{2}$ but is absent in $\mathrm{CeCu}_{2} \mathrm{Si}_{2}$, which supports the idea that magnetic order and $\mathrm{SC}$ are not directly related [6]. For $\rho_{0}$
$>50 \mu \Omega \mathrm{cm}$, the depression of $T_{\mathrm{c}}^{\text {bulk }}$ of $\mathrm{CeCu}_{2} \mathrm{Si}_{2}$ with increasing $\rho_{0}$ becomes much weaker, and $\mathrm{SC}$ survives even when $\rho_{0}$ is of the order of the Ioffe-Regel limit 34]. However, the large $\rho_{0}$ could be due to the effect of Kondo holes [35, 36], and thus is no longer a good indication of the level of disorder.

The sensitivity of $\mathrm{SC}$ in $\mathrm{CeAu}_{2} \mathrm{Si}_{2}$ to nonmagnetic disorder also allows of an explanation for the transition at $T^{*}$ observed in $\mathrm{CeAu}_{2} \mathrm{Si}_{2}$ (self). It is theoretically demonstrated that when coupled to quantum critical fluctuations, disorder can induce regions of local order or even long range order in the host phase [37]. Indeed, a recent experimental study shows that nonmagnetic $\mathrm{Cd}$ impurities in $\mathrm{CeCoIn}_{5}$ nucleate magnetic regions even when global magnetic order is suppressed and bulk SC is restored by pressure, which is ascribed to the local competition between magnetism and SC [38]. As shown above, the emergence of the transition at $T^{*}$ may be related to a putative QCP, and almost coincides with the establishment of bulk $\mathrm{SC}$ in $\mathrm{CeAu}_{2} \mathrm{Si}_{2}(\mathrm{Sn})$. It is possible that near the disorder sites in $\mathrm{CeAu}_{2} \mathrm{Si}_{2}(\mathrm{Sn}), \mathrm{SC}$ is locally destroyed and regions of competing order are formed. With increasing disorder, these regions are expected to grow in size and start to overlap. As a result, above a certain level of disorder, $\mathrm{SC}$ is destroyed completely and long range order develops, as in $\mathrm{CeAu}_{2} \mathrm{Si}_{2}$ (self).

\section{CONCLUSION}

In summary, we have studied the effect of disorder on the pressure-induced heavy fermion superconductor $\mathrm{CeAu}_{2} \mathrm{Si}_{2}$ through the comparison of high-pressure results from single crystalline samples with two different $\rho_{0}$ values. It is found that, with the increase of $\rho_{0}$, both the pressure range for $\mathrm{SC}$ and the maximum $T_{\mathrm{c}}$ are reduced, although the critical behaviors near the magneticnonmagnetic boundary and the delocalization of Ce $4 f$ electrons under pressure are essentially unaffected. The bulk $T_{\mathrm{c}}$ dependence on $\rho_{0}$ near the optimum pressure for $\mathrm{SC}$ is very similar to that of $\mathrm{CeCu}_{2} \mathrm{Si}_{2}$, and is consistent with the pair breaking effect by nonmagnetic disorder. These results not only provide evidence for unconventional $\mathrm{SC}$ in $\mathrm{CeAu}_{2} \mathrm{Si}_{2}$ under pressure, but also suggest that the two $\mathrm{Ce}_{2} \mathrm{Si}_{2}(X=\mathrm{Cu}$ or $\mathrm{Au})$ compounds share a common pairing mechanism. Finally, for the sample with a higher $\rho_{0}$ value, a new phase transition appears at $T^{*}$ below $T_{\mathrm{M}}$, which is probably related to an order that competes with SC. In this respect, the clarification of the nature of the transition at $T^{*}$, which may help to elucidate the pairing mechanism, is worth pursuing in future studies. 


\section{ACKNOWLEDGEMENT}

We acknowledge technical assistance from M. Lopes, and financial support from the Swiss National Science Foundation through Grant No. 200020-137519.

* Electronic address: Zhi.Ren@unige.ch

[1] G. Knebel, D. Aoki, and J. Flouquet, Comptes Rendus Physique 12, 542 (2011).

[2] D. Jaccard, K. Behnia, and J. Sierro, Phys. Lett. A 163, 475 (1992).

[3] F. M. Grosche, S. R. Julian, N. D. Mathur, and G. G. Lonzarich, Physica 223-224B, 50 (1996).

[4] N. D. Mathur, F. M. Grosche, S. R. Julian, I. R. Walker, D. M. Freye, R. K. W. Haselwimmer, and G. G. Lonzarich, Nature (London) 394, 39 (1998).

[5] H. Hegger, C. Petrovic, E. G. Moshopoulou, M. F. Hundley, J. L. Sarrao, Z. Fisk, and J. D. Thompson, Phys. Rev. Lett. 84, 4986 (2000).

[6] Z. Ren, L. V. Pourovskii, G. Giriat, G. Lapertot, A. Georges, and D. Jaccard, Phys. Rev. X 4, 031055 (2014).

[7] F. Steglich, J. Aarts, C. D. Bredl, W. Lieke, D. Meschede, W. Franz, and H. Schafer, Phys. Rev. Lett. 43, 1892 (1979).

[8] O. Stockert, J. Arndt, E. Faulhaber, C. Geibel, H. S. Jeevan, S. Kirchner, M. Loewenhaupt, K. Schmalzl, W. Schmidt, Q. Si, and F. Steglich, Nat. Phys. 7, 119 (2011).

[9] A. T. Holmes, D. Jaccard, and K. Miyake, Phys. Rev. B 69, 024508 (2004).

[10] P. W. Anderson, J. Phys. Chem. Solid 11, 26 (1959).

[11] Y. Dalichaouch, M. C. de Andrade, D. A. Gajewski, R. Chau, P. Visani, and M. B. Maple, Phys. Rev. Lett. 75, 3938 (1995).

[12] S. K. Tolpygo, J.-Y. Lin, M. Gurvitch, S. Y. Hou, and J. M. Phillips, Phys. Rev. B 53, 12454 (1996).

[13] A. P. Mackenzie, R. K. W. Haselwimmer, A. W. Tyler, G. G. Lonzarich, Y. Mori, S. Nishizaki, and Y. Maeno, Phys. Rev. Lett. 80, 161 (1998).

[14] B. J. Powell and R. H. McKenzie, Phys. Rev. B 69, 024519 (2004).

[15] M. Nicklas, F. Steglich, J. Knolle, I. Eremin, R. Lackner, and E. Bauer, Phys. Rev. B 81, 180511(R) (2010).

[16] P. Monthoux, D. Pines, and G. G. Lonzarich, Nature
(London) 450, 1177 (2007).

[17] A. T. Holmes, D. Jaccard, G. Behr, Y. Inada, and Y. Onuki, J. Phys.:Condens. Matter 16, S1121 (2004).

[18] A. T. Holmes, Ph.D. thesis, Université de Genève, 2004 http://archive-ouverte.unige.ch/unige:284.

[19] B. Cornut and B. Coqblin, Phys. Rev. B 5, 4541 (1972).

[20] Y. Nishida, A. Tsuruta, and K. Miyake, J. Phys. Soc. Jpn. 75, 064706 (2006).

[21] N. Tateiwa, Y. Haga, T. D. Matsuda, S. Ikeda, T. Yasuda, T. Takeuchi, R. Settai, and Y. Onuki, J. Phys. Soc. Jpn. 74, 1903 (2005).

[22] D. Jaccard, H. Wilhelm, K. Alami-Yadri, and E. Vargoz, Physica 259-261B, 1 (1999).

[23] G. Seyfarth, A.-S. Ruetschi, K. Sengupta, A. Georges, D. Jaccard, S. Watanabe, and K. Miyake, Phys. Rev. B 85, 205105 (2012).

[24] A. T. Holmes, D. Jaccard, and K. Miyake, J. Phys. Soc. Jpn. 76, 051002 (2007).

[25] B. Bellarbi, A. Benoit, D. Jaccard, J. M. Mignot, and H. F. Braun, Phys. Rev. B 30, 1182 (1984).

[26] K. Miyake and O. Narikiyo, J. Phys. Soc. Jpn. 71, 867 (2002).

[27] A. A. Abrikosov and L. P. Gor'kov, Sov. Phys. JETP 12, 1243 (1961).

[28] P. I. Larkin, Sov. Phys. JETP Lett. 2, 130 (1965).

[29] A. J. Millis, S. Sachdev, and C. M. Varma, Phys. Rev. B 37, 4975 (1988).

[30] R. J. Radtke, K. Levin, H.-B. Schüttler, and M. R. Norman, Phys. Rev. B 48, 653(R) (1993).

[31] M. B. Maple, J. W. Chen, S. E. Lambert, Z. Fisk, J. L. Smith, H. R. Ott, J. S. Brooks, and M. J. Naughton, Phys. Rev. Lett. 54, 477 (1985).

[32] H. Q. Yuan, F. M. Grosche, M. Deppe, C. Geibel, G. Sparn, and F. Seglich, New J. Phys. 6, 132 (2004).

[33] E. Vargoz, Ph.D. thesis, Université de Genève, 1998.

[34] A. T. Holmes, D. Jaccard, H. S. Jeevan, C. Geibel, and M. Ishikawa, J. Phys.:Condens. Matter 17, 5423 (2005).

[35] J. M. Lawrence, J. D. Thompson, and Y. Y. Chen, Phys. Rev. Lett. 54, 2537 (1985).

[36] D. Jaccard, E. Vargoz, K. Alami-Yadri, and H. Wilhelm, Rev. High Pressure Sci. Technol. 7, 412 (1998).

[37] A. J. Millis, D. K. Morr, and J. Schmalian, Phys. Rev. Lett. 87, 167202 (2001).

[38] S. Seo, X. Lu, J.-X. Zhu, R. R. Urbano, N. Curro, E. D. Bauer, V. A. Sidorov, L. D. Pham, T. Park, Z. Fisk, and J. D. Thompson, Nat. Phys. 10, 120 (2014). 